\title{
Epidemiological Study on the Oral State in Pregnant Women
}

\author{
C. Mlaouah ${ }^{1}$, S. Ziada ${ }^{2}$, M. Chebil ${ }^{3}$, I. Blouza ${ }^{4}$ \\ ${ }^{1}$ DDS, Private Clinic \\ ${ }^{2}$ DDS, Department of Conservative Dentistry and Endodontics, Faculty of Dental Medicine of Monastir \\ ${ }^{3}$ Associate professor, Department of Fixed Prosthodontics, Faculty of Dental Medicine of Monastir \\ ${ }^{4}$ Professor, Department of Medicine and Oral Surgery,Military Hospital of Tunis
}

\begin{abstract}
Introduction: Pregnancy plays a preponderant role in the development of oral and dental pathologies by the action of steroid sex hormones on the different systems of the maternal organism and in particular the immune, vascular and secretory systems, as well as on the gingival flora. and oral. Objective: Our goal was to compare oral status in pregnant versus non-pregnant women to determine whether or not there is a relationship between hormonal disruption during pregnancy and increased incidence of oral disease. Material and methods: We conducted an epidemiological survey. This investigation took place in the gynecology department of the military hospital of Tunis. The sample consists of 200 women divided into two groups: A test group consisting of 100 pregnant women and a control group consisting of 100 non-pregnant women. Exclusion criteria were: Age <19 years, Pathology requiring antibiotic prophylaxis for periodontal probing, such as cardiac pathology, General pathology that may affect periodontal status (such as unbalanced diabetes), females toothless or with less than 8 teeth, outside the third molars. This is a prospective, comparative study between two groups, based on a form developed with an epidemiologist. The survey was carried out using a questionnaire completed by one and the same person during a clinical intraoral examination and relating to the marital status, general pathologies of the patient and her hygiene habits. Periodontal status was assessed for each patient, and we used the bleeding index, plaque index, gingival index, and community index for assessing CPITN periodontal care needs: Community Periodontal Index of Treatment Needs). The data is captured and analyzed using SPSS software version 20. The statistical test used is Pearson's Chi 2 to study the significance of the difference between independent variables. The materiality threshold is set at $5 \%$. Results: The results showed that there is a level of oral hygiene oscillating between the moderate and the bad, a bad condition of the dentition in pregnant women. The physiological state of pregnancy aggravates the situation, so the impact of pregnancy on the periodontal state is manifested by an increase in the gingival index, the bleeding index and the depth of the pockets. These results also showed that pregnancy requires both obstetric and oral followup.
\end{abstract}

Keywords: pregnancy, gum score, oral impact, tooth decay

\section{Introduction}

Pregnancy is a period of significant transformation of the female body, namely, hormonal, neurological, cardiovascular, hematologic. The hormonal changes that a woman undergoes during the gestation period, can promote the appearance of oral pathologies. These attacks can be mucous, dental or salivary.The objective of our study was to locate the level of oral status in pregnant women compared to non-pregnant women, in order to determine whether there is pregnancy involvement on the condition oral.

\section{Materials and Methods}

Method :This is a prospective, comparative study between two groups, based on a form developed with an epidemiologist.

Material :The clinical examination was performed using a mirror, a tongue depressor, a portable light source and a WHO periodontal probe, graduated and equipped with a 0.5 spherical tip. $\mathrm{mm}$ in diameter that is used for exploration of periodontal pockets.

Survey form: The survey was carried out using a questionnaire completed by one and the same person during a clinical endobuccal examination and relating to the marital status, general pathologies of the patient and her hygiene habits.

Several parameters were studied in the test group and the control group to evaluate the dento-periodontal state. Among these parameters we used the CAD index, bleeding index (Loe and Silness, 1964), the plaque index, the gingival index (Loe, 1967) [1], Inter-radicular lesion (Hamp index). Dental mobility (Mühelemann index), and the community index for periodontal care needs assessment CPITN: Community Periodontal Index of Treatment Needs).

The mouth is divided into sextants defined by the numbers of the teeth:18-14, 13-23,24-28,33-43, 38-34,44-48

This system only applies to ten teeth:11,16-17, 26-27,41,3637, 46-47.

Each tooth was scored in six locations: vestibular, mesiobuccal, disto-vestibular, lingual, mesio-lingual, disto-lingual and each sextant was scored according to its highest score.The scores from 0 to 4 are recorded by sextant with the following criteria: 0 = No signs listed below. 1 = bleeding gingival bleeding, no pocket, no calculus, no plaque. $2=$ the pocket depth does not exceed $3 \mathrm{~mm}$ but also when calculating scale or other plaque retention factors. $3=4$ to 5 $\mathrm{mm}$ pocket, $4=6 \mathrm{~mm}$ or more pocket.The sextants are then graded to undertake the following therapeutic attitudes: $0=$

Volume 6 Issue 12, December 2017 


\section{International Journal of Science and Research (IJSR) \\ ISSN (Online): 2319-7064 \\ Index Copernicus Value (2016): 79.57 | Impact Factor (2015): 6.391}

no treatment $(0), \mathrm{I}=$ dental hygiene instruction (code 1$), \mathrm{II}=$ oral hygiene instruction + descaling (code 2 and code 3 ), III $=$ dental hygiene instruction + descaling + complex treatment (code 4)

Data Analysis: The data is entered and analyzed using SPSS Version 20 software. The statistical test used is Pearson's Chi 2 to study the significance of the difference between independent variables. The materiality threshold is set at $5 \%$.

\section{Results}

\subsection{Periodontal characteristics}

\subsubsection{Plaque Index}

There was no statistically significant difference in plaque index $(\mathrm{p}=0.552) .95 \%$ of pregnant women have dental plaque compared to $93 \%$ of non-pregnant women (Fig. 1).

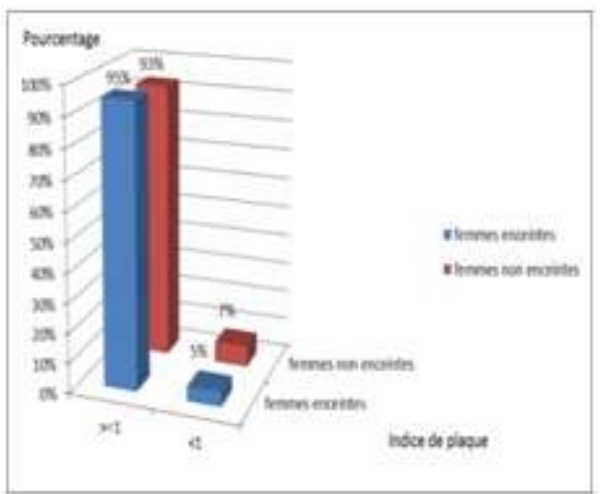

Figure 1: Distribution according to the plaque index

\subsubsection{Bleeding Index}

$98 \%$ of pregnant women have gingival bleeding compared to $81 \%$ of non-pregnant women. There is a statistically significant difference between the two groups $(p=0.0001)$ (Figure 2).

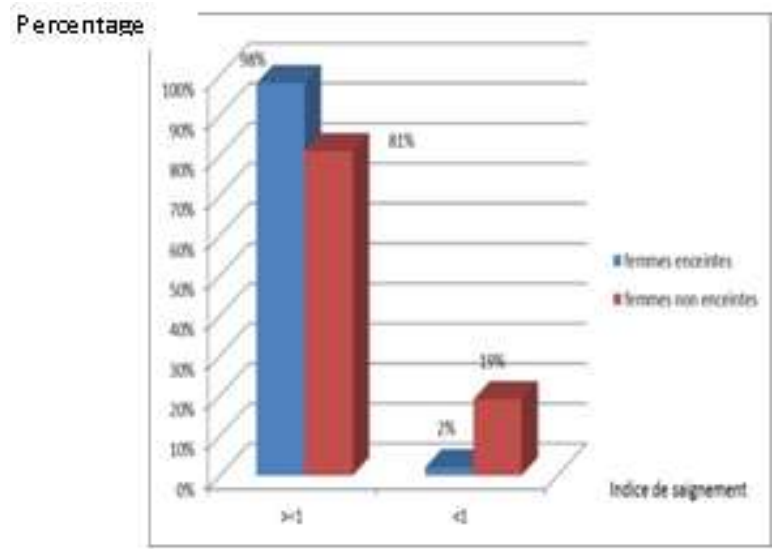

Figure 2: Distribution according to the bleeding index

\subsubsection{Gingival Index}

The results of our study revealed that the gingival index varies between 1 and 3. However, the group of pregnant women shows a gingival index varying between 2 and 3, greater than that of the group of non-pregnant women, respectively $98 \%$ and $81 \%$; this difference is statistically significant $(\mathrm{p}=0.001)$ (Figure 3$)$.

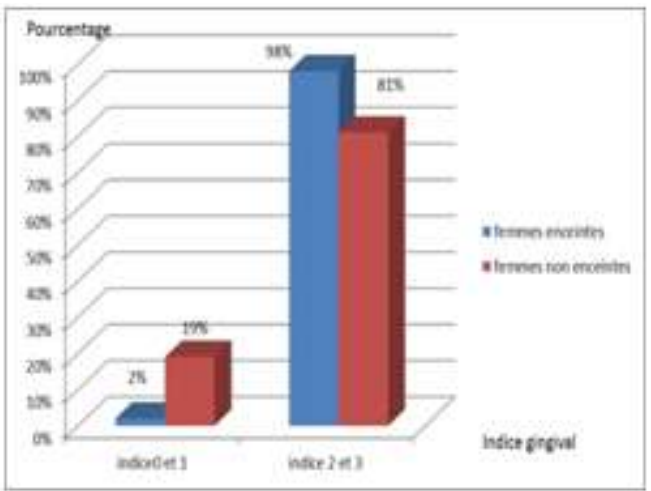

Figure 3: Distribution according to the gingival index

\subsubsection{CPITN}

The results of our study show that the CPITN varies between 1 and 4. The index 0 was not found in any of the women of the two groups. $80 \%$ of the pregnant women have an index varying between 3 and 4 against only $46 \%$ of the nonpregnant women. The difference is statistically significant ( $p$ $=0.001)$ (Figure 4).

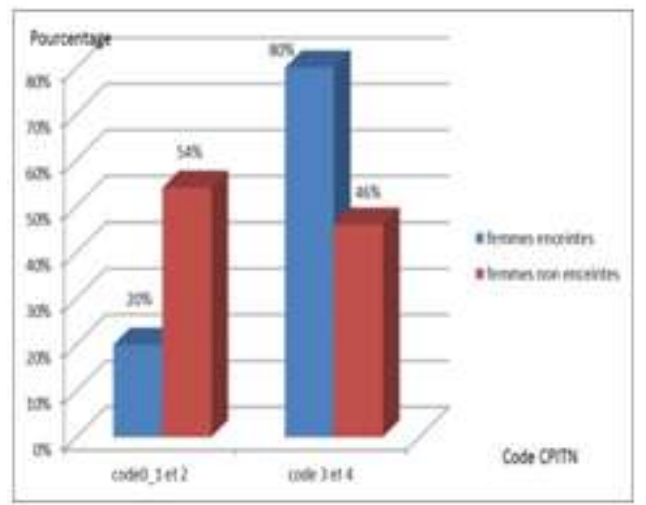

Figure 4: Distribution according to the CPITN

\subsubsection{Gingival Growth}

Regarding gingival growth, there is a statistically significant difference between the two groups $(p=0.01)$. $44 \%$ of pregnant women had a gingival increase compared to $21 \%$ of non-pregnant women (Figure 5)

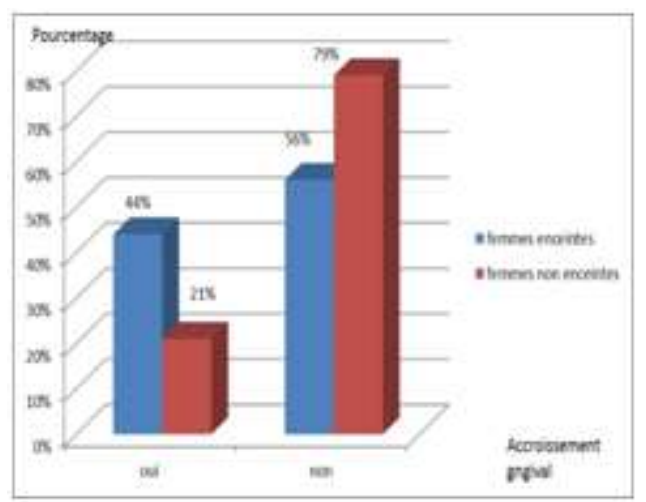

Figure 5: Distribution according to gingival growth 


\section{International Journal of Science and Research (IJSR)}

ISSN (Online): 2319-7064

Index Copernicus Value (2016): 79.57 | Impact Factor (2015): 6.391

\subsection{Dental Disorders}

\subsubsection{Dental Caries}

$85 \%$ of pregnant women have at least one decayed tooth, compared with $65 \%$ of non-pregnant women (Figure 6). The difference is statistically significant $(\mathrm{p}=0.01)$

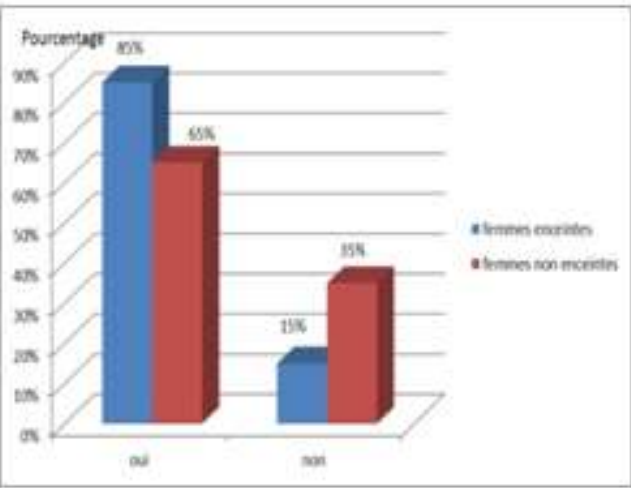

Figure 6: Distribution according to the presence or not of decayed teeth.

\subsubsection{Absence of teeth}

$54 \%$ of pregnant women have at least one missing tooth compared to $40 \%$ of women in the control group (Figure 7) .This difference is statistically significant $(\mathrm{p}<0.05)$.

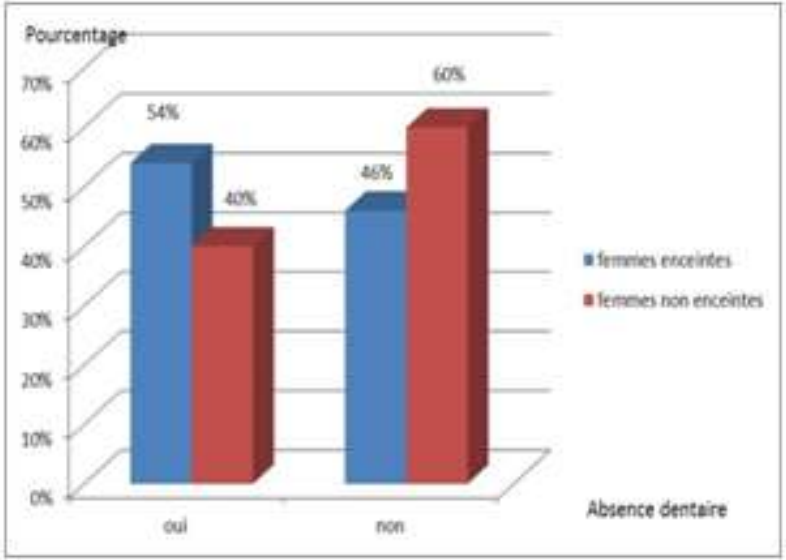

Figure7: distribution according to dentalabsence.

\subsubsection{Dental Mobility}

The results of our survey showed that the majority of women had no dental mobility (fig8). The difference is not statistically significant $(\mathrm{p}=0.651)$.

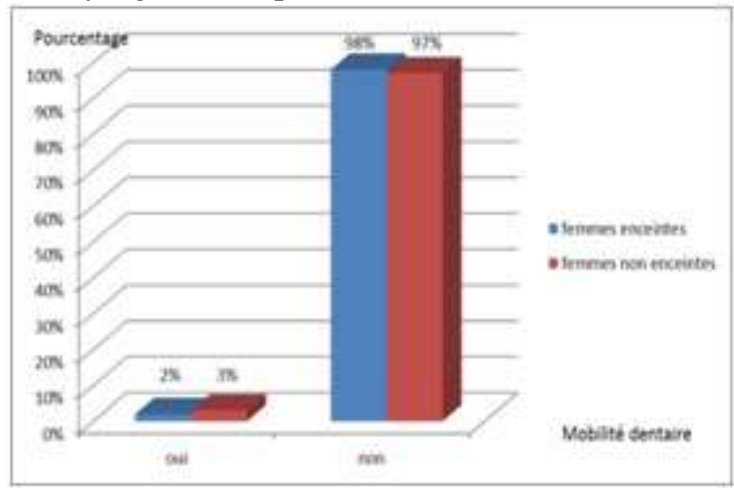

Figure 8: Répartitionselonlamobilitédentaire

\subsubsection{Interradicular Lesions}

The results of our survey showed that $98 \%$ of pregnant women and $97 \%$ of non-pregnant women had no interradicular lesions (Figure 9). The difference is statistically insignificant $(\mathrm{p}=0.651)$

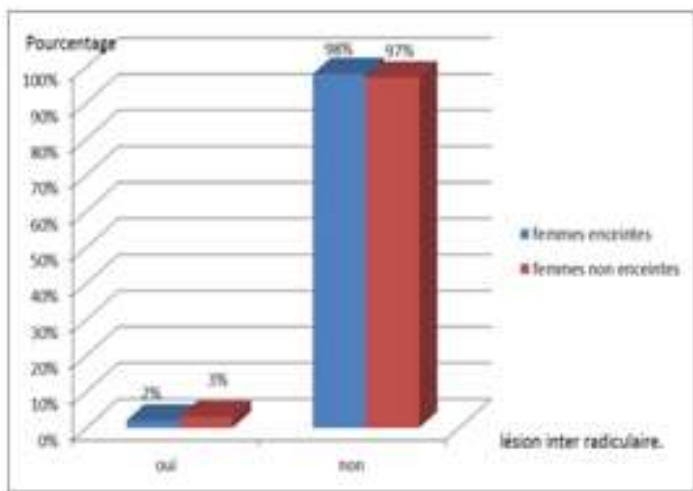

Figure 9: Distribution according to interradicular lesions

\section{Discussion}

During pregnancy, hormonal disturbances can influence the oral state in pregnant women, so it is important to highlight the implication of this factor (pregnancy) on the oral state, in order to motivate the pregnant women about the need for care before and during gestation

The study was conducted on a population of 100 pregnant women and 100 non-pregnant women. $27 \%$ of pregnant women and $31 \%$ of non-pregnant women belong to the socio-professional category of managers. $62 \%$ of pregnant women and $55 \%$ of non-pregnant women were of urban origin. Only $2 \%$ of non-pregnant women presented a notion of smoking. $87 \%$ of pregnant women did not consult a dentist during their pregnancy. At the Bichat-Gynecology Talks in 1991, it was reported that only $5 \%$ of pregnant women spontaneously consult the dentist during pregnancy. [2]

Marchi and colleagues have shown that $65 \%$ of women in California did not consult a dentist during pregnancy [3]. Many patients avoid seeing a dentist for fear of affecting their baby (Strafford et al., 2008). [4]In our study all patients reported brushing their teeth at least once a day. Indeed, $68 \%$ of pregnant women brush their teeth 2 and 3 times a day. In spite of a good frequency of brushing, one finds gingival bleeding. The results showed that $98 \%$ of pregnant women had gingival bleeding compared to $81 \%$ of nonpregnant women, but this relationship is statistically significant. These results are in agreement with the study by Nuhmann and Annan (1998) who conducted a study of pregnant women in Ghana, found $89 \%$ of pregnant women with bleeding compared to $61 \%$ of control women. [5]

Regarding the gingival index, the results also showed that the test group has a gingival index significantly greater than that of the control group, respectively, $98 \%$ and $81 \%$. This confirms the findings reported by several authors: (Ziskin et al., 1993, Main et al., 1949, Hilming et al., 1952, Chaput et al., 1953) who encountered only $12 \%$ of healthy gingiva. [6] In addition, Samant et al. in 1976 showed that there is a significant increase in the severity of gingivitis during

Volume 6 Issue 12, December 2017 


\section{International Journal of Science and Research (IJSR) \\ ISSN (Online): 2319-7064}

Index Copernicus Value (2016): 79.57 | Impact Factor (2015): 6.391

pregnancy. [7] According to Loé and Silness in 1963, the prevalence of gingivitis is $100 \%$. [8] Another epidemiological survey in Morocco showed the same results. [6] This inflammation can be explained by the immunological changes caused by the increase of hormonal levels during pregnancy, this finding is that of Charles and Bellavoir in 1989, which showed that progesterone, the polypeptide hormones and certain glycoproteins associated with pregnancy have immunosuppressive properties. In addition, polynuclearchemotaxis, phagocytosis and antibody response are affected in the presence of a high level of these hormones. (Lopatin et al., 1980). [8] This gingivitis acquires these characters from the second trimester and does not regress until after delivery. The aggravation of this gingivitis is caused mainly by the increase of the rate of the sex hormones and by its effects on the micro vascularization. Indeed, progesterone acts on the gingival vascular permeability and induces hair proliferation and neoformation according to Lindhe and Brånemark. [6,8]In addition, hormonal changes cause disruption of folate metabolism. This results in an imbalance in tissue maturation and fragility of the epithelial tissue (Dreizen et al.). [8,9]

The level of hygiene has been studied also by the determination of the plaque index. Our results showed that there is no significant difference between two groups, $95 \%$ of pregnant women have plaque versus $93 \%$ of non-pregnant women. These results are consistent with the results of an epidemiological survey in Casablanca [6] and findings found by RaberDurlacher and other authors in 1994 where they demonstrated in a study that the amount of plaque was similar during pregnancy and postpartum. This can be explained by the lack of oral hygiene. [10]. In fact, gingival inflammation promotes the existence of niches, inaccessible to brushing and favorable to the colonization of anaerobic bacteria. As for the capillary proliferation, the consequence is to increase the gingivorragies. The pregnant woman, for fear of causing bleeding, brushes her teeth less well, which promotes the accumulation of plaque. It is therefore not surprising to see in these patients significant deposits of plaque.

In this study, there was no evidence of a significant relationship between gingivitis and plaque index. For Carranza et al. (1988), the ratio of gingival inflammation to the amount of plaque is narrower after delivery than during pregnancy. [6]

In this regard, Raber-Durlacher et al. Suggested that following plaque accumulation, the development of gingival inflammation is directly related to the physiological alterations associated with pregnancy. [10]. We move on to the impact of pregnancy on the deep periodontium, we studied the mobility, the inter-radicular lesion, and the code CPITN.

Women in both groups were assessed according to the community periodontal index of treatment needs, the results are statistically significant. $80 \%$ of pregnant women have an index CPITN between 3 and 4 against $46 \%$ of non-pregnant women; therefore the majority of pregnant women have pockets that require complex periodontal treatment, except $20 \%$ of this group require scaling and hygiene motivation.
These results are consistent with Davenport et al. who report a prevalence of $4 \mathrm{~mm}$ pockets of $44 \%$ in a group of British women evaluated immediately after delivery. [11] In addition, Miyazaki et al. (1991) showed that pregnant women had significant periodontal pockets that were deeper than non-pregnant women. Changes have been interpreted to suggest that the increase in pocket depth during pregnancy was caused by gingival swelling, rather than by periodontal destruction. [12] Indeed, Brochut and Cimasoni hypothesize that if there is no destruction of the deep periodontium it is that the inflammatory reactions related to pregnancy are ephemeral. [2] This finding is also confirmed by Loe and Silness (1963), Arafat (1974), Samant et al. (1976). [8] For this purpose, Lapp, Lewiss, Billman, in 2003 used several methods to test in vitro the hypothesis that progesterone levels could inhibit the production of certain matrix metalloproteases that are responsible for periodontal destruction. This may help explain why gingivitis is not necessarily characterized by progression of periodontitis. [13]

For tooth mobility, Cohen et al. (1971), (Hage et al., 1996), found from their studies that horizontal mobility is more important in pregnant women than non-pregnant women. [14] Rateitschak et al. reported a significant change in mobility during and after pregnancy. They explained that mobility depends on the degree of vascularization and vascular volume of the periodontal membrane, and that sex hormones can increase the permeability of the periodontal vasculature. [15] M. SIDQUI et al. (2002) found, following an epidemiological study of the impact of pregnancy on the deep periodontium, that only the degree of tooth mobility was significantly higher in the test group than in the control group ( $\mathrm{p}<0.001)[16]$.

However In our study the majority of women in both groups had no dental mobility. The relationship between the two groups is not statistically significant.

Regarding interradicular lesions, the same results are found. Thus, the gravid state seems to affect only the marginal periodontium. On the other hand, there has been a focus on tooth decay, which is a multifactorial infectious disease, whose development is related to the interaction between hormonal, behavioral and socio-economic influences. It has been hypothesized that pregnancy could increase carious risk, through changes in the composition of saliva (Wool 2002). [17] Indeed, during pregnancy, progesterone tends to lower the concentrations plasma levels of bicarbonate. This would help reduce the salivary concentration of $\mathrm{HCO} 3$ which decreases the buffering capacity of saliva. The development of carious lesions is thus accentuated by the marked increase in streptococcus mutans at the end of pregnancy (Laine et al., 1986). [8] Tooth decay requires some time to develop, while pregnancy is a short period, it is likely that decay develops from initial carious lesion. Nevertheless, it is interesting to study the prevalence of caries during pregnancy because caries often leads to painful and stressful situations during this period, with negative effects on the quality of life of pregnant women. [17]

An epidemiological study of 504 pregnant women showed a $39 \%$ frequency of oral pain during pregnancy primarily

\section{Volume 6 Issue 12, December 2017}




\section{International Journal of Science and Research (IJSR) \\ ISSN (Online): 2319-7064}

Index Copernicus Value (2016): 79.57 | Impact Factor (2015): 6.391

caused by decay and these pains affect the subject's normal activities much more than headaches and pelvic pain. $[18,19]$

Some studies have evaluated the frequency of caries during pregnancy, they showed values between $47 \%$ and $69 \%$. In a Pakistani cohort study of 1152 pregnant women (mean age 26.5 years), nearly $47 \%$ of women had at least one decayed tooth. A study in Hungary showed that $69 \%$ of postpartum mothers needed one or more restorations. [20] Vergnes found that more than $50 \%$ of postpartum women had at least one decayed tooth. [16]

In our study, the results showed that most pregnant women had carious lesions, $85 \%$, and $65 \%$ for the non-pregnant women group, this is a significant difference. This significant difference may be related to the age of the patients, since the age of women varies between 19 and 42 years and non-pregnant women are older than pregnant women, $37 \%$ of non-pregnant women aged over 35 years against $20 \%$ of pregnant women. In fact, some studies have shown that caries is associated with lower age groups. One explanation might be that young women are more likely to develop inappropriate eating behavior such as nibbling. Another hypothesis is that young adults are less likely to regularly consult a dentist. [17].

For dental absence, it was found that $54 \%$ of pregnant women had missing teeth compared to $40 \%$ of non-pregnant women. This relationship is statistically significant. The prevalence of tooth loss among Ugandan women is $35.7 \%$. [11]

Indeed, some studies have shown that increased tooth loss during pregnancy is linked to aggravating factors such as parity (Rundgren and Osterberg 1987, Halling and Bengtsson 1989, Christensen et al. al., 2008). [4].The results of the survey we conducted show the need: to inform pregnant women of the risks due to pregnancy; $\bullet \cdot \bullet$ a health education to encourage them to consult more with the medical practitioner and especially for a motivation to good oral hygiene.

\section{Conclusion}

The aim of our study was to locate the level of oral status in pregnant women compared to non-pregnant women, in order to determine whether there is pregnancy involvement on the condition oral. The results showed that there is a level of oral hygiene oscillating between the moderate and the bad, a bad condition of the dentition in pregnant women. The physiological state of pregnancy aggravates the situation, so the impact of pregnancy on the periodontal state is manifested by an increase in the gingival index, the bleeding index and the depth of the pockets. These results also showed that pregnancy requires both obstetric and oral follow-up.

\section{References}

[1] CharonJ.Parodontiemédicale( $2{ }^{\text {ème }}$ édition).Paris: $C d P$ ,2010.
[2] DechercqJ,DeVillersA,BiserteM.Pathologiesbuccode ntairesdesfemmesenceintesetfumeuses.ChirDentFr200 0;922/923:13-22.

[3] MarchiKS,Fisher-OwensSA,WeintrauJA,YuZ, BravemanPA.Mostpregnantwomenincaliforniado not receive dental care: findings from a population-based study. PublicHealthRep2010;125:831-42.

[4] RussellSL,IckovicsJR,YaffeeRA.Parity\&untreatedde ntalcariesinuswomen.JDentRes2010;89:1091-6.

[5] NuamahI,AnnanBD.Periodontalstatusandoralhygiene practiceof pregnant and non pregnant women. East Afr MedJ1999;75:712-4.

[6] Hermas S, Salah-Eddine A, Sidqui Metal. Répercussiondel'étatgravidiquesurlestissusparodontaux .Ref Gynecol Obstet1999; 6:340-6.

[7] SamantA,MalikCP,ChabraSK,DeviPK.Gingivitisan dperiodontaldiseaseinpregnancy.JPeriodontal1976;47: 415-8.

[8] HageG,DavarpanahM,KebirM,TecucianuJF,Askar iN.Grossesseetétatparodontal:revuedelittératureaspectscliniques.JParodontolImplOrale 1996;15:37987.

[9] DreizenS, LevyBB,BernickS.Studiesonthebiologyof theperiodontiumof marmosets.VIII.Theeffectoffolic aciddeficiencyonthemarmosetoralmucosa.JDentRes 19 70;49:616-20.

[10] Raber-

DurlacherJE,vanSteenbergenTJ,vanDerVel denU,deGraaffJ, AbrahamInpijnL.Experimentalgingivitisduringpregnan cyandpostpartum:clinical,endocrinological,and microbiologicalaspects.JClinPeriodontol1994; 21:549-48

[11] WanderaMN,EngebretsenIM,OkulloIet al.Sociodemographicfactorsrelatedtoperiodontalstatusandtoothl ossof pregnant womenin Mbaledistrict,Uganda.BMCOralHealth2009;9:18.

[12] MiyazakiH,YamashitaY,ShirahamaRet al.Periodontalconditionof pregnantwomenassessedbyCPITN.JClinPeriodontol19 91;18:751-4.

[13] LappCA,LewisJB,LohseJE,DicksonDP.Theeffectsof progesteroneonmatrixmetalloproteinasesinculturedhum an gingivalfibroblasts.JPeriodontal2003;74:277-88.

[14] HAGE G., DAVARPANAH M., KEBIR M., TECUCIANU J.F., ASKARI N. Grossesse et état parodontal : Revue de littérature aspects cliniques. J. Parodontol., 1996, 15, n 4. , P. 379 - 387.

[15] 15. RateitschakKH.Tooth mobility changes in pregnancy.J Periodontal Res 1967;2:199-206.

[16] M.SIDQUI, M.LAZRAQ, S.HERMASS, K.AMINE, J. KISSA (Faculté de Médecine Dentaire de Casablanca).Répercussions de la grossesse sur les tissus parodontaux. lecourrrier du dentiste ,15 MARS 2002.

[17] Vergnes JN. Epidémiologiedesmaladiesbuccodentairechezlafemmeenceinte:Facteursde risqueet association avecaccouchementprématuré [Thèse]. Toulouse:UniversitéPaulSabatier,FacultédeChirurgie Dentaire,2011.

[18] Oliveira BH, Nadanosvsky P. The impact of oral pain on quality of life during pregnancy in low-income Brazilian women.JOrofacPain2006;20:297-305. 


\title{
International Journal of Science and Research (IJSR) \\ ISSN (Online): 2319-7064 \\ Index Copernicus Value (2016): 79.57 | Impact Factor (2015): 6.391
}

[19] Wandera MN, Engebretsen IM, Rwenyonyi CM, Tumwine J. Period ontal status, tooth loss and selfreported periodontal problems effects on oral impacts on daily performances, OIDP, in pregnant women in Uganda: across- sectional study. Health Qual Life Outcomes 2009;7:89.

\section{Annex}

Investigation Form
[20] Vergnes JN, KaminskiM, Lelong Netal. Frequency and risk indicators of tooth decay among pregnant women in France: a cross-sectional analysis. PLoS One, 2012;7:e33296.

Date :

Name first name :

Date of birth :

origin :

Socio economic level :

1-profession : no occupation Others (specify).......

2- level of study : primary Secondary university

\section{Antecedents :}

1- family : Medical: Hypertension .... diabetes... allergy...

2-- Personnel: Medical-Surgical : Hypertension. diabetes. .caesarean

..... allergy......

others(specify)

Bucco dental consultation with the dentist before pregnancy : yes.....no

General state :

Term of pregnancy: ...... weeks .....days

gravidity :...

Parity :....

Tobacco : yes... no...

$\begin{array}{lllll}\text { Consultation with the dentist during pregnancy: } & \text { yes } \ldots & \text { no } & \ldots & \\ \text { Brushing teeth Frequency : } 1 \text { time /day } \ldots . . & 2 \text { times /day } \ldots & & 3 \text { times/day... }\end{array}$

\section{Intraoral examination}

1-Hygiene: poor average fair/good

2-Presence of gingival growth: yes no

3-Bleeding index : $0 \quad 1 \quad 2 \quad 2 \quad 3$

4-Plaqueindex : $0 \quad 1 \quad 2 \quad 3$

5-gingival index : $0 \quad 1 \quad 2 \quad 3$

\section{6- CPITN:}

B
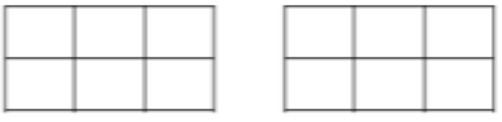

P

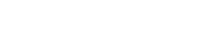

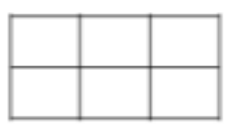

L

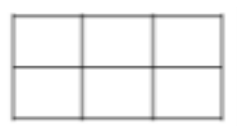

B
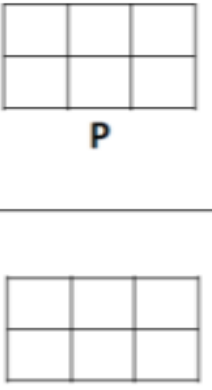

L

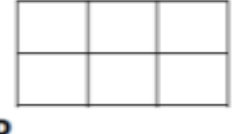

Evaluation of periodontal treatment needs:

7- Examendentaire :

Caries

Mobility

interradicular lesions according to HAMP

Number of missing teeth

\begin{tabular}{|c|c|c|c|c|c|c|c|c|c|c|c|c|c|c|c|c|}
\hline Teeth & 18 & 17 & 16 & 15 & 14 & 13 & 12 & 11 & 21 & 22 & 23 & 24 & 25 & 26 & 27 & 28 \\
\hline Caries & & & & & & & & & & & & & & & & \\
\hline Mobility & & & & & & & & & & & & & & & & \\
\hline
\end{tabular}

Volume 6 Issue 12, December 2017

\author{
www.ijsr.net
}

Licensed Under Creative Commons Attribution CC BY 
International Journal of Science and Research (IJSR)

ISSN (Online): 2319-7064

Index Copernicus Value (2016): 79.57 | Impact Factor (2015): 6.391

\begin{tabular}{|c|l|l|l|l|l|l|l|l|l|l|l|l|l|l|l|l|}
\hline Missingteeth & & & & & & & & & & & & & & & & \\
\hline $\begin{array}{c}\text { Interradicular } \\
\text { Lesions }\end{array}$ & & & & & & & & & & & & & & & \\
\hline
\end{tabular}

\begin{tabular}{|c|r|r|r|r|r|r|r|r|r|r|r|r|r|r|r|r|}
\hline Teeth & 38 & 37 & 36 & 35 & 34 & 33 & 32 & 31 & 41 & 42 & 43 & 44 & 45 & 46 & 47 & 48 \\
\hline Caries & & & & & & & & & & & & & & & & \\
\hline Mobility & & & & & & & & & & & & & & & & \\
\hline Missingteeth & & & & & & & & & & & & & & & & \\
\hline $\begin{array}{c}\text { InterradicularL } \\
\text { esions }\end{array}$ & & & & & & & & & & & & & & & \\
\hline
\end{tabular}

Volume 6 Issue 12, December 2017

www.ijsr.net

Licensed Under Creative Commons Attribution CC BY 